Cómo citar este artículo: Ortiz, A., Sánchez, J. y Sánchez, I. (2015, enero-julio). Los modelos pedagógicos desde una dimensión psicológica-espiritual. Rev. Cient. Gen. José María Córdova 13(15), 183-194

\title{
Los modelos pedagógicos desde una dimensión psicológica-espiritual*
}

Recibido: 11 de noviembre de 2014 - Aceptado: 10 de enero de 2015

The Pedagogical Models from a Psychological and Spiritual Dimension

Les modèles pédagogiques dans ses dimensions psychologique et spirituelle

Os modelos pedagógicos por meio de uma dimensão psicológica e espiritual

Alexander Ortiz Ocaña ${ }^{a}$

Jorge Oswaldo Sánchez Buitrago ${ }^{b}$

Iván Manuel Sánchez Fontalvo ${ }^{c}$

* Artículo de investigación asociado al proyecto MODEPED: identificación, caracterización y elaboración del modelo pedagógico de las instituciones públicas del Caribe colombiano, financiado por la Universidad del Magdalena.

a Universidad del Magdalena, Santa Marta, Colombia. Doctor en Ciencias Pedagógicas, Universidad Pedagógica de Holguín, Cuba; Doctor Honoris Causa en Iberoamérica, Consejo Iberoamericano en Honor a la Calidad Educativa (CIHCE), Lima, Perú; Magíster en Gestión Educativa en Iberoamérica, CIHCE, Lima, Perú; Magíster en Pedagogía Profesional, Universidad Pedagógica y Tecnológica de la Habana; Contador Público; Licenciado en Educación. Recibió el premio a la excelencia educativa 2007 y 2008 otorgado por el CIHCE con sede en Lima, Perú. Mejor pedagogo novel de Cuba en el año 2002. Ha realizado asesorías pedagógicas, talleres y conferencias en Cuba, México, Brasil, Ecuador, Venezuela y Panamá, así como en múltiples instituciones educativas y universidades de Colombia. Docente de planta de tiempo completo de la Universidad del Magdalena, Santa Marta, Colombia. Investigador del Doctorado en Ciencias de la Educación de Rude Colombia. Coordinador del grupo de investigación GIDECOM: Desarrollo y evaluación de competencias. Contacto: alexanderortiz2009@gmail.com

b Universidad del Magdalena, Santa Marta, Colombia. Docente de planta de la Universidad del Magdalena —Cade: Rude Colombia—. Doctor en Educación (Universidad de Salamanca, España); Magíster en Desarrollo Educativo y Social (Universidad Pedagógica Nacional, Centro Internacional de Educación y Desarrollo Humano CINDE); Licenciado en Educación con especialidad en Administración Educativa, Universidad Católica de Manizales. Miembro de los grupos de investigación CEMPLU (Calidad educativa en un mundo plural) y GRACE (Grupo de Análisis de la Cultura Escolar). Contacto: joswaldosanchez@gmail.com

c Universidad del Magdalena, Santa Marta, Colombia. Docente de planta tiempo completo de la Universidad del Magdalena, adscrito a la Facultad de Ciencias de la Educación; Doctor en Calidad 
Resumen. Se analizan los modelos pedagógicos desde una dimensión psicológica, haciendo una breve descripción del modelo pedagógico conductista (Skinner), el modelo pedagógico constructivista (Piaget) y la teoría socio-histórico-cultural (Vygotsky) como modelo pedagógico. Sin embargo, existen diversas propuestas pedagógicas holísticas y ecológicas derivadas de nuevas epistemologías. En este sentido concluimos que desde el modelo bio-pedagógico Maturana propone una biología del amor como sustento ontológico y epistemológico de la Pedagogía del Amor, que brinda otras opciones holísticas, sistémicas y configuracionales de comprender el aprendizaje humano, muy diferentes a las concepciones conductista, constructivista e histórico cultural.

Palabras clave: aprendizaje humano, biología del amor, conductismo, constructivismo, modelo pedagógico, pedagogía del amor, teoría histórico-cultural, teorías del aprendizaje.

Abstract. In this article the pedagogical models analyzed from a psychological dimension, making a brief description of the pedagogical model behavior (Skinner), the constructivist teaching model (Piaget) and historical-sociocultural (Vygotsky) theory as a pedagogical model. However, there are several proposed teaching holistic and ecological derived from new epistemologies. In this sense we conclude that from the model bio-pedagogical Maturana proposed a biology of love as ontological and epistemological basis of the pedagogy of love, which provides holistic alternatives, systemic and configurational understand learning human, very different from the behaviorist, constructivist and historical conceptions cultural.

Key words: behaviorism, biology of love, constructivism, cultural theory, human learning, pedagogical model, the pedagogy of love, theories of learning.

Résumé. Modèles pédagogiques sont analysées à partir d'une dimension psychologique, avec une brève description du modèle pédagogique comportementale (Skinner), le modèle constructiviste pédagogique (Piaget) et la théorie socio-historico-culturelle (Vygotski) comme un modèle d'enseignement. Cependant, il ya plusieurs propositions holistiques et écologiques de l'éducation nouvelle épistémologie. En ce sens, nous concluons que le modèle bio-pédagogique Maturana propose une biologie de l'amour comme fondement ontologique et épistémologique de la pédagogie de l'amour, qui donne d'autres options holistiques, systémiques et configurationnels de comprendre l'apprentissage humain, des concepts très différents de comportement, culture constructiviste et historique.

Mots clés. apprentissage humain, biologie de l'amour, behaviorisme, constructivisme, modèle pédagogique, pédagogie de l'amour, théorie historico-culturelle, théories de l'apprentissage.

Resumo. Modelos pedagógicos são analisados a partir de uma dimensão psicológica, com uma breve descrição do modelo pedagógico comportamental (Skinner), o modelo construtivista pedagógica (Piaget) e da teoria sócio-histórico-cultural (Vygotsky) como um modelo de ensino. No entanto, existem várias propostas de educação holística e ecológicas de nova epistemologia. Nesse sentido, concluímos que a partir do modelo bio-pedagógica Maturana propóe uma biologia do amor como base ontológica e epistemológica da Pedagogia do Amor, que dá outras opçôes holísticas, sistêmicas e configuracionais para entender a aprendizagem humana, conceitos muito diferentes de comportamento, cultura construtivista e histórico.

Palavras-chave. aprendizagem humana, biologia amor, o behaviorismo, construtivismo, modelo pedagógico, pedagogia do amor, a teoria histórico-cultural, as teorias da aprendizagem. 


\section{Introducción}

Todo proceso educativo tiene un método, una guía o un eje, que encamine y contribuya al desarrollo más adecuado del ejercicio de formar. A esto le llamamos modelo pedagógico.

Cada teoría ha privilegiado en ello algún o algunos de los aspectos; aun así subyace a todas ellas una postura como individuo y como ser social y cultural. A partir de esta concepción del ser humano se elaboran las teorías pedagógicas. En este sentido toda teoría pedagógica es una teoría política. (De Zubiría, 2007, p. 39).

Precisamente, la pedagogía es una ciencia cuyas fronteras muestran su dinámica entre la sociedad y el pensamiento humano. Un modelo pedagógico es un plan teórico y práctico de estrategias autónomo que poseen el docente y las instituciones educativas para desarrollar el proceso de formación de sus estudiantes.

El modelo pedagógico se caracteriza por la articulación de nociones como: currículo, pedagogía, didáctica, formación, educación, enseñanza, aprendizaje y evaluación; pero además contribuye a la configuración de procesos como la práctica y la teoría. Para la configuración e identificación de un modelo pedagógico es importante, según Coll (1991), responder cinco preguntas esenciales: ¿para qué enseñar?, ¿qué enseñar?, ¿cómo enseñar?, ¿cuándo enseñar?, y ¿qué, cómo y cuándo evaluar?

La primera pregunta se refiere a las intencionalidades, a los propósitos que se pretenden, todos estos encaminados en un sentido a través de la formulación de objetivos y ligados a los contenidos que responden a la segunda pregunta. Los contenidos son las temáticas comprendidas en el currículo, su secuencia y el orden cronológico de los temas, es decir, en qué jerarquía se organizan estos contenidos. En la tercera pregunta es evidente la importancia de una metodología de enseńanza con la utilización de estrategias pedagógicas y didácticas determinadas para el tratamiento de las temáticas contempladas en la primera pregunta. La cuarta pregunta tiene que ver con la relación de los protagonistas del contexto educativo (maestro, estudiante y saber) y sus funciones correspondientes, con el fin de relacionarse con la cuarta pregunta. Por último ¿qué, cómo y cuándo evaluar?, es la finalización de un proceso que permite la retroalimentación y el cumplimiento de los propósitos y objetivos plasmados en la primera pregunta (para qué enseñar).

\section{El modelo pedagógico conductista}

Skinner asegura que la limitación de los conductistas anteriores a él consistió en considerar solo el antes del comportamiento, para tratar de construir una psicología basada en el estímulo-respuesta, siendo necesario, según él, tener presente lo que ocurre después, lo que cambia la probabilidad de que lo ocurrido vuelva a repetirse, para poder hacer una adecuada explicación del comportamiento y de la influencia que tiene el ambiente en él. 
Sobre esto, Luzoro ${ }^{1}$ le pregunta a Maturana: ¿de qué manera es coincidente o contradictorio con su punto de vista este análisis funcional que hace Skinner de la relación entre comportamiento y ambiente?

Lo que dice Skinner en términos de lo que ocurre en las interacciones de los organismos, ya sea entre ellos o con el medio, ocurre. No cabe ninguna duda que si uno considera, como él dice, las consecuencias de la conducta en el análisis del curso histórico de estas en un ser humano, uno descubre el fenómeno del refuerzo. Lo prueban claramente sus procedimientos experimentales. Maturana (2003) con eso no tiene ninguna contradicción. "Nunca tiene uno problemas con la praxis. Los problemas surgen con las explicaciones de la praxis, pues es allí donde surgen proposiciones diferentes" (p. 205). De esta manera, Maturana no tiene discrepancia con lo que Skinner revela en su trabajo experimental. Su divergencia con Skinner comienza en el intento explicativo. Concuerda con Skinner cuando él dice, por ejemplo, que no son válidas las explicaciones que usan nociones como mente, intención o deseo, pero no concuerda con él cuando se queda con la praxis solamente. El problema con las explicaciones en términos mentales no se encuentra en que uno no pueda encontrar coherencias operacionales de valor predictivo con ellas, sino en que no son adecuadas para la generación de los fenómenos conductuales cuando uno reconoce que los organismos son sistemas determinados por sus configuraciones.

De hecho solo es posible explicar lo que pasa con el cambio conductual en la historia de un organismo, cuando se pone atención a su dinámica estructural, ya que es la deriva estructural del organismo en sus interacciones con el medio lo que hace posible la participación de las consecuencias del comportamiento en el curso del comportamiento. (Maturana, 2003, p. 206)

Luzoro precisa que Skinner define estímulo como cambio en el ambiente que altera la probabilidad del comportamiento y le pregunta a Maturana ¿cuál es la diferencia entre el estímulo así concebido y la noción de perturbación?

La diferencia no es poca si se concibe el estímulo como un suceso ambiental que yo observo y describo haciendo referencia a las consecuencias que tiene en la conducta del organismo en términos probabilísticos. "Lo que un observador describe como circunstancia ambiental al describir el estímulo no es necesariamente lo que el organismo encuentra en su interacción” (Maturana, 2003, p. 206).

Maturana (2003) llama perturbación a lo que de hecho el organismo encuentra en la interacción y que el observador no ve a menos que use al organismo como indicador de ella. En otras palabras, estímulo es lo que el observador ve incidir sobre el organismo, y perturbación es lo que el organismo admite como encuentro que gatilla en él una transformación configuracional en la que se conserva su adaptación y organización. Skinner no hace ni puede hacer esta distinción porque ella solo surge de considerar al organismo como un sistema determinado por sus configuraciones, y él no hace eso. Sin embargo, la distinción entre estímulo y perturbación es irrelevante si uno tiene en cuenta el determinismo configuracional del organismo al explicar la conducta. Como Skinner no lo hace, él puede decir, sin contradecirse, que cualquier circunstancia del

${ }^{1}$ Este diálogo fue realizado en Santiago, en enero de 1987 y fue publicado inicialmente en la Revista Chilena de Psicología, 9(1), 1987, 77-86 y luego en el libro Desde la Biología a la Psicología, 2003, 195-216.. 
ambiente puede participar en el curso de las interacciones de un organismo y transformarse en estímulo. Esto, porque de hecho "lo que ocurre en la praxis del condicionamiento operante es que el estímulo adquiere una connotación de perturbación al quedar definido en el encuentro operacional del organismo con el medio que le da validez" (Maturana, 2003, p.206).

En estas circunstancias, siguiendo a Maturana (2003), lo que ocurre en el encuentro organismo-medio no es probabilístico, sino determinista en el ámbito del determinismo configuracional de la interacción. Para Skinner, que no tiene en cuenta la configuración estructural del organismo y solo mira al cambio conductual en el intento de predecir su curso, la situación es probabilística como reflexión en cuanto a la ignorancia que se tiene de ella como sistema determinado por sus configuraciones. De este modo, la descripción del condicionamiento operante como una situación que altera la probabilidad de la repetición de una conducta solo refleja la limitación predictiva del observador, y no la naturaleza del fenómeno que es determinista.

Maturana ha afirmado que a él le gusta la obra de Skinner. Le gusta porque lo encuentra serio en su no dejarse atrapar por intentos explicativos que no hacen referencia a los mecanismos generativos de los fenómenos que a él le interesan. El que en este proceso Skinner se quede con la praxis que él domina, a Maturana le parece que se debe a que Skinner está interesado en la manipulación de la conducta más que en la explicación. Es por esto que Maturana ve a Skinner más como a un tecnólogo que como un científico, en su intención.

Skinner sigue fielmente la tradición de la civilización occidental que dice: conocer es controlar, y que la manera de saber lo que es algo realmente está en que pueda controlarse o reproducir experimentalmente, dice Luzoro. Pero según Maturana hay una diferencia entre explicar y controlar.

Skinner piensa eso en la tradición que ve a la investigación científica como asociada al control y la predicción de los fenómenos en el mundo; y Maturana (2003) ve la explicación científica no asociada a la predicción o el control, sino asociada, en general, a la explicación como reformulación del fenómeno a explicar y, en particular, a la "proposición de un mecanismo que genere el fenómeno a explicar en el contexto de la satisfacción de ciertas condiciones que constituyen el criterio de aceptación de las explicaciones científicas" (p.207).

La noción de manipulación, o sea, de control, no forma parte de la explicación científica ni del hacer ciencia, sino del hacer tecnología. Tecnología y ciencia requieren de emociones distintas: pasión explicativa en el hacer ciencia, y pasión productiva o pasión de control en el hacer tecnología.

En nuestra cultura la pasión productiva o controladora es central en muchas dimensiones de la vida cotidiana individual o comunitaria. De esta manera, los seres humanos occidentales estamos inmersos en las manipulaciones productivas de todo tipo, físicas, culturales, etc. Pertenecemos a una cultura básicamente tecnológica. Maturana (2003) comprende a Skinner en su preocupación manipulativa aunque no la comparte, y le gusta la seriedad con la cual él funciona evitando la confusión de dominios y el quedarse atrapado en proposiciones explicativas que apuntan fuera del ámbito científico, como por ejemplo "cuando se usan nociones de procesos mentales para explicar la conducta” (Maturana, 2003, p. 207).

Skinner, sin embargo, continúa Maturana (2003), no ofrece una explicación científica de la conducta. Él desarrolla tecnologías manipulativas de la conducta, pero no la explica científica- 
mente, porque no propone mecanismos generativos; y no lo puede hacer, porque no considera a los organismos como sistemas determinados por sus configuraciones. Sabe que lo son, pero no lo tiene en cuenta. Entonces, "se queda en el juego de la manipulación del determinismo estructural de los organismos a través de las perturbaciones ambientales, aunque no las vea como tales sino como estímulos" (p. 208).

Skinner explica las concatenaciones históricas de las conductas pero no explica científicamente el fenómeno del comportamiento en el ámbito del comportamiento. Cuando Skinner propone que el lenguaje surge en las concatenaciones de contingencias de refuerzo está a un paso de decir que el lenguaje se configura en las coordinaciones conductuales consensuales, pero no lo dice, y en este sentido no explica el fenómeno del comportamiento.

El libro de Skinner dedicado al lenguaje lo llama Verbal Behaviour, porque propone explicar el lenguaje en términos de contingencia de refuerzo. Él, de hecho, lo desea en ese ámbito al decir que es en las contingencias de refuerzo de las interacciones de las personas donde surge lo que pasa con el fenómeno del lenguaje. Con esto, lo que dice del lenguaje es cómo se daría su fluir en la convivencia como fenómeno de coordinaciones conductuales, pero no dice que el lenguaje es un dominio fenoménico configurado en la recursión de las coordinaciones conductuales consensuales, y al no hacerlo, no tiene en cuenta la dinámica que genera ese fenómeno, pero sí analiza la dinámica en la cual ese fenómeno se manipula.

Skinner dice que el lenguaje básicamente es una forma de comportamiento, que lo distintivo es que las consecuencias que tiene ese comportamiento están mediatizadas por el escuchante. De esta manera, Luzoro no ve que haya una diferencia entre esa concepción y la concepción del lenguaje entendido como la recursión de coordinaciones conductuales consensuales, esbozada por Humberto Maturana.

Por supuesto, no hay una diferencia en el sentido que Maturana también dice que el lenguaje es una forma de comportamiento que involucra tanto al que habla como al que escucha. Lo que pasa es que Maturana dice qué tipo particular de forma de comportamiento es el lenguaje y cómo se genera y, por lo tanto, qué consecuencias tiene ese modo de generación, como por ejemplo, la generación del metadominio y el surgimiento del objeto.

De hecho hay todo un conjunto de otras cosas que Skinner no toca, y, en criterio de Maturana (2003), no toca porque no analiza el lenguaje como un fenómeno que genera un espacio particular conductual, sino que analiza simplemente una manipulación de la conducta cuando destacar que es un comportamiento. Él está preguntándose ¿cómo es que las personas dicen las cosas que dicen y en las circunstancias que dicen esas cosas? Él piensa en términos de contingencia de refuerzo, y no ve lo propio del lenguaje como un modo particular de conducirse en las contingencias de refuerzo.

Como el lenguaje pertenece al ámbito de las coordinaciones conductuales, pertenece al ámbito de la coderiva, y, las contingencias de refuerzo en las convivencias son circunstancias de coderiva. El resultado es que Skinner apunta a la coderiva, pero como no atiende al mecanismo de la coderiva de sistemas determinados por sus configuraciones, no ve lo que pasa en el lenguaje como una coderiva en la recursión de las coordinaciones conductuales, y se queda en las contingencias de refuerzos (Maturana, 2003). 
Para Skinner lo central viene a ser el refuerzo en función de lo que le pasa al organismo, y no lo que pasa entre los organismos en las concatenaciones de refuerzos.

\section{El modelo pedagógico constructivista}

El paradigma constructivista comienza a gestarse en la década de los años veinte en el siglo XX en los trabajos del eminente psicólogo y epistemólogo suizo Jean Piaget, quien casi no necesita presentación.

Piaget nace en Suiza en 1896, se formó inicialmente como biólogo y desde muy pequeño mostró intereses científicos muy marcados. A modo de ejemplo se puede mencionar que a los once ańos publica su primer artículo científico.

Piaget constituye un antecedente muy significativo en la historia de la educación con respecto a una biología del conocimiento. La obra de Piaget es conocida y estudiada por muchos psicólogos y educadores. Dos de sus concepciones fundamentales son la de asimilación y la de acomodación.

Dentro de la pregunta general por el conocimiento, Piaget (1976) se detiene frente a las diferencias que se observan en los nińos en cuanto al desempeño cognitivo: su trabajo junto a Simon le había mostrado claramente que hay problemas que los niños son incapaces de resolver en ciertas etapas de su desarrollo, aun cuando se le instruya explícitamente para hacerlo. Este resultado es interpretado por Piaget como una prueba fehaciente de que la resolución de los problemas depende del desarrollo de ciertas estructuras cognitivas. Además, la epistemología propuesta y desarrollada por Piaget sustenta que las estructuras de la cognición humana se desarrollan a partir de una génesis de alguna estructura anterior: por medio de procesos de transformación constructivas, las estructuras más simples van siendo incorporadas en otras de orden superior. "Es en este sentido que esta epistemología es llamada genética, no en el sentido de la disciplina biológica que estudia a los genes, como algunas veces se confunde” (Rosas y Sebastián, 2010, p.12).

En este punto tal vez sea conveniente hacer una breve síntesis de las etapas del desarrollo planteadas por Piaget (1976):

1. Etapa sensorio motriz (cero a dos años), cuyo máximo logro es la adquisición de la función simbólica o capacidad de representar el mundo externo por medio de símbolos.

2. Etapa pre operacional (dos a siete ańos), cuyo máximo logro es la preparación, a partir del ejercicio activo del uso de símbolos, para la adquisición de las "operaciones mentales", las que son descritas por Piaget como estructuras cognitivas que le permiten al individuo operar en el ambiente de manera lógica y reversible.

3. Etapa de las operaciones concretas (siete a doce años), caracterizada por el ejercicio de la lógica en la acción del individuo con los objetos de su entorno.

4. Etapa de las operaciones formales (a partir de los doce años), caracterizada por la posibilidad del individuo de operar en el ambiente de manera hipotético-deductiva, aun en ausencia de experimentación práctica. (Rosas y Sebastián, 2010, p. 27)

Por ser esta la faceta de la obra del autor más conocida (si no la única, en muchos casos), hemos preferido dejarla para el final y solo como ilustrativa del problema de los contenidos de la cogni- 
ción. Es importante notar en la descripción que hace Piaget sobre los estadios del desarrollo infantil, que las estructuras cognitivas tienden a diferenciarse máximamente en las distintas edades marcadas por las etapas. Los principios de organización y adaptación, sin embargo, son los que le dan identidad al desarrollo, a pesar de las transformaciones cualitativas de las estructuras a las diferentes edades.

Ángel Pérez Gómez le pregunta a Maturana si está de acuerdo con el planteamiento que hacía Piaget, de que los instintos son adaptaciones cognitivas hereditarias.

Con esta expresión en términos generales, sí, dice Maturana. No estoy de acuerdo con la visión histórica que eso implica [...] pero es cierto, el instinto..., es eso pero, el instinto es una cosa un poco distinta, en el sentido de que es una conducta que surge separada de la historia particular del organismo que la exhibe, y entonces puede surgir adecuada a la circunstancia que está o, inadecuada a la circunstancia que está, porque surge con independencia de la historia particular..., ahora, "normalmente uno se mueve no, en cualquier parte, uno se mueve en un espacio con ciertas características en las cuales, claro, esos instintos son efectivos, no son conductas adecuadas" (Maturana, 2003, p.136).

Por otro lado, en el diálogo sostenido entre Jorge Luzoro García y Humberto Maturana sobre psicología, Luzoro le pregunta a Maturana sobre los procesos de asimilación y acomodación definidos por Piaget.

Maturana ve a Piaget como un gran pensador e investigador en lo psicológico y en lo biológico, que tiene en cuenta, lo que le parece a él, es también su pregunta: ¡en qué consiste el fenómeno del conocer como fenómeno biológico?

Los biólogos durante mucho tiempo han dejado esta pregunta relegada a un ámbito que no es el biológico, el ámbito de la filosofía. Piaget no lo hace. Al contrario, él toma en serio el carácter biológico del fenómeno del conocer; y Maturana (2003) piensa que lo que él hace en ese sentido es magnífico. Sin embargo, Maturana tiene ciertas discrepancias con Piaget, que surgen de ciertas diferencias en sus puntos de partida, y que hacen que sus caminos sigan cursos finales algo distintos. Por ejemplo, al usar las expresiones de asimilación y acomodación, Piaget indirectamente está aceptando la opción de que el organismo capta la realidad externa como parte de su mecanismo de subsistencia. Y Maturana piensa diferente.

El vivir ocurre como una deriva estructural en la que la conversación de la adaptación o correspondencia con el medio es una condición constituida de la existencia de un organismo, ya que en su ausencia se desintegra. De modo que el organismo, en su vivir, sigue un curso u otro de transformación estructural, según el camino de conservación de la adaptación que cada instante se le da. (Maturana, 2003, p. 203)

Entonces, para Maturana (2003) no hay asimilación y no hay una acomodación en los términos que uno corrientemente como observador podría describir cuando piensa: "he ahí un problema", "una dificultad" y he aquí que este animalito o este niño, que yo estoy mirando, está acercándose a su solución en un proceso de transformación que solo puedo entender como dirigido hacia un estado final. Por esto podré decir cuando llegue él a resolverlo que ha habido un proceso de acomodación o asimilación a circunstancias ambientales definidas por ese estado final hacia el cual él se va. 
Maturana (2003) dice que "la aproximación a un estado final que uno observa en el desarrollo de una conducta es una consecuencia histórica de un proceso de deriva, y no una transformación activa” (p. 203). En un sentido estricto, la descripción de lo que ocurre en el cambio conductual como asimilación y acomodación a una realidad independiente, es literatura y pertenece a los comentarios del observador.

A diferencia de Piaget, Maturana no plantea su explicación del conocer como una interpretación de las muchas posibilidades de ese fenómeno. Su proposición la plantea como la explicación ontológica, es decir, como la presentación de las condiciones constitutivas el fenómeno biológico que connotamos cuando hablamos de conocer.

Las discusiones siempre se plantean en un sentido y otro según el uso de las palabras. Por eso Maturana ha especificado lo que dice, caracterizando el fenómeno del conocer con el modo como reconocemos cotidianamente la presencia del conocimiento. "Conocimiento es lo que un observador le adscribe a otro organismo en un ámbito particular, cuando observa en este una conducta que él o ella considera efectiva en ese ámbito" (Maturana, 2003, p.204).

Por esto mismo, Maturana considera que su explicación es ontológica, ya que revela las condiciones constitutivas del fenómeno del conocer al mostrar cómo surge cualquier conducta efectiva como parte del vivir de un organismo en el dominio en que ella se observa.

En otras palabras, Maturana (2003) dice que en cada caso particular que usamos la palabra conocimiento, el fenómeno que connotamos con ella es conducta efectiva de un organismo en un dominio particular de acoplamiento de configuraciones, y que como tal es parte del vivir de aquel en ese dominio. Lo que Maturana propone, por lo tanto, no es una interpretación, porque no está dando cuenta del origen de las consecuencias del fenómeno del conocer, sino de las condiciones que lo constituyen. Es ontología, no hermenéutica.

\section{La teoría socio-histórico-cultural como modelo pedagógico}

Vygotsky nació en 1896 en el seno de una familia judía, en Rusia. Tuvo una infancia muy estimulada en lo cultural por sus padres, caracterizada por el aprendizaje desde pequeño de varios idiomas, la formación básica con un tutor de alto nivel intelectual y la educación formal en un gymnasium judío. Durante su infancia y adolescencia adquirió en estos contextos su amor por el teatro, la poesía y la literatura. De hecho los trabajos intelectuales desarrollados durante su adolescencia y juventud estuvieron orientados principalmente hacia la literatura y el lenguaje.

Su formación universitaria se desarrolló en Moscú, en dos universidades de manera simultánea: la de Moscú y la de Shanyavskii. En la primera se graduó en leyes en 1917. En la segunda, institución no oficial formada por alumnos y profesores que habían sido expulsados de la universidad oficial por el Ministerio de Educación zarista, Vygotsky obtuvo una acabada formación en filosofía, historia, psicología y literatura, fruto de la presencia de buena parte de los mejores profesores de Moscú en ese momento, en dicha universidad.

Una vez graduado, vuelve a su ciudad natal para dedicarse a una gran cantidad de tareas de diverso orden. Hasta su partida a Moscú en 1924, Vygotsky hace clases en varios establecimientos secundarios, de formación de profesores y de educación de trabajadores; participa de diversos gru- 
pos de discusión literaria y teatral; publica artículos en revistas y periódicos; lee en profundidad a filósofos como Spinoza, Hegel y Marx y a psicólogos como Pavlov, Freud y James. Junto a todo lo anterior redacta en esta época diversos ensayos acerca de estas materias.

En 1924, Vygotsky hizo una brillante presentación en el II Congreso de neuropsicología en Leningrado, luego es invitado a trabajar al Instituto de Psicología de Moscú. Vygotsky desarrolló su trabajo en esta ciudad durante la última década de su vida, diez años de una febril actividad que incluyó fundamentalmente la docencia universitaria, la dirección de numerosos proyectos de investigación y el trabajo con personas que tenían algún tipo de discapacidad (perceptual, mental o por problemas de aprendizaje).

El fruto de esta empresa fue la formulación entre 1928 y 1931, en conjunto con A. R. Luria, de la teoría histórico-cultural, la cual ofrece un marco explicativo unificado para procesos psicológicos elementales y superiores. Vygotsky enfatiza no obstante, que no hay nada "sobrenatural" en los procesos superiores, ya que en términos genéricos estos están formados por la integración de dos o más procesos elementales (Rosas y Sebastián, 2010).

Vygotsky (1979) alude a cuatro grandes criterios que permiten diferenciar de modo general entre procesos psicológicos elementales y superiores (Rosas y Sebastián, 2010):

a) El primero de ellos dice relación con la vía de control de unos y otros. En tanto los procesos psicológicos elementales son controlados automáticamente por el entorno, los procesos superiores se caracterizan por una autorregulación por parte del individuo, la cual descansa en la creación y utilización de estímulos artificiales que se transforman en los gatillantes inmediatos de la conducta.

b) Directamente ligado con lo anterior, se puede afirmar que los procesos psicológicos superiores tienen un alto nivel de regulación consciente, o por lo menos lo necesitaron en su origen (aun cuando luego, por vía de la utilización repetida del mecanismo este se haya "automatizado", requiriendo posteriormente menor cantidad de recursos conscientes).

c) Un tercer aspecto que caracteriza diferencialmente a los procesos psicológicos superiores es su naturaleza lo que llevaría a la aparición de los procesos superiores, sino la participación de los seres humanos en contextos de relaciones sociales directas en pequeños grupos o diadas.

d) En la concepción vigotskiana, "el control voluntario, la realización consciente y la naturaleza social de los procesos superiores presuponen la existencia de herramientas psicológicas o signos, que pueden ser utilizados para controlar la actividad propia y de los demás" (Wertsch, 1985, p. 44). La incorporación de estas herramientas psicológicas (de origen social) es lo que se define como mediación semiótica, proceso que corresponde a la característica principal de funcionamiento intelectual propiamente humano, dentro del enfoque de la psicología socio-histórica de Vygotsky. (1979, p. 33)

Esta noción de mediación semiótica se asienta en la analogía entre herramienta material y signo, que Vygotsky (1979) establece y precisa. Vygotsky sigue y amplía el análisis de Marx y Engels (1973) acerca del rol del trabajo en la organización de la vida social propiamente humana. En dicho análisis se afirma que la aparición de la herramienta material, que permite instaurar la noción de trabajo (en tanto transformación productiva de la naturaleza), es crucial en la historia de la conformación de las sociedades humanas. A través de la herramienta el ser humano puede dejar de depender de la naturaleza, para orientar su acción hacia ella de manera intencional. 
Para Vygotsky (1979) existe un período inicial en el desarrollo del niño que está fuertemente marcado por la línea natural. Desde los cero a los dieciocho a veinte meses se puede observar el desarrollo de una inteligencia práctica, perfectamente análoga a la de los chimpancés, la cual incluye tanto capacidades perceptivas como motrices que le permiten resolver exitosamente tareas complejas que están dentro de su campo perceptivo y de los límites de su envergadura corporal.

La aparición del lenguaje, sin embargo, implica para Vygotsky (1979; 1981) un cambio cualitativo en el desarrollo de los procesos psicológicos observados hasta ese momento: con este se abre la capacidad de un verdadero control del individuo sobre su entorno físico. Así como para los simios de los experimentos de Köhler $(1967$; 1972), los cuales enfrentados a una resolución de tareas prácticas conscientes en alcanzar objetos agradables (normalmente comida) vivencian una reconfiguración de su representación de la situación al incorporar una herramienta material como un palo, dentro de su campo visual; la incorporación por parte de los niños en tareas análogas implica una reconfiguración de la situación total. El lenguaje implica tanto la posibilidad de planeación de la acción, por parte del niño, incorporando así herramientas materiales y psicológicas que están más allá del campo visual; como el control voluntario de dicha acción; y el control posible de otras personas presentes en la situación a través de la utilización comunicativa del lenguaje.

\section{Conclusiones}

Existen dos formas diferentes de orientar un proceso formativo, asociadas a las dos formas de responder la pregunta por el mundo en que vivimos. Existen dos miradas diferentes de lo que sucede en la vida cotidiana de nuestros estudiantes, que no podemos soslayar. Como resultado de la educación en la que están inmersos nuestros estudiantes, existen dos concepciones diferentes acerca de las transformaciones que se generan en ellos, la mirada racionalista, dogmática, mecanicista, reduccionista o fragmentaria; y la mirara socio-crítica, dialéctica, compleja, holística, ecológica o configuracional.

En la primera mirada, el profesor cree que existe ante sus ojos un universo franqueable al que tiene acceso inmediato, y en este sentido actúa como el poseedor de un conocimiento absoluto que puede ser transmitido de manera directa e inmutable al estudiante. En la otra mirada, el profesor no se cree un erudito que posee todo el poder cognoscitivo, sino que admite la posibilidad de que el estudiante configure su propio mundo y su propia realidad a partir de sus configuraciones presentes y actuales, generadas en su biopraxis. En esta forma de enseñar el profesor es un mediador, un guía que ayuda al estudiante a comprender el mundo que configura en su biopraxis cotidiana. El primer camino es el de la objetividad sin paréntesis, el segundo camino es el de la objetividad entre paréntesis. La primera forma de ver la educación es fragmentaria, un pensamiento mecánico y dogmático, la segunda forma es holística e integradora, un pensamiento configuracional. Por ejemplo, en el modelo biopedagógico de Maturana (1992; 1994; 1995; 1996; 1999; 2003), el ser humano está guiado por la conservación vital: principios morales trascendentales (moral natural), en este sentido se aleja de la propuesta de Piaget de un sujeto guiado por el imperativo categórico: deducción de principios morales a partir de principios trascendentes, y tampoco coincide con el ser humano de Vygotsky, guiado por la noción de progreso: principios morales racionales, pero históricamente situados. 
Para Vygotsky (1979) la finalidad de la educación es la internalización de herramientas semióticas y compartir comunidad de sentido, para Piaget (1976) es el desarrollo del juicio moral y del pensamiento científico como operaciones formales, y para Maturana (2003) la finalidad de la educación es brindar amor, es decir, promover la aceptación del otro como legítimo otro en la convivencia, considerando el amor como la emoción básica que funda lo humano y lo social, como condición biológica de todos los seres humanos. Es por ello que su modelo es bio-pedagógico, basado en el amor. Maturana (2003) propone una biología del amor como sustento ontológico y epistemológico de la Pedagogía del Amor, que brinda otras opciones holísticas, sistémicas y configuracionales de comprender el aprendizaje humano, muy diferentes a las concepciones conductista (Skinner), constructivista (Piaget) e histórico cultural (Vygotsky).

\section{Referencias}

1. Coll, C. (1991). Psicología y currículo. Editorial Paidós. Madrid. España.

2. De Zubiría, J. (2007). Los Modelos Pedagógicos: Hacia una pedagogía dialogante. Bogotá: Editorial Magisterio.

3. Köhler, W. (1967). Psicología de la configuración. Introducción a los conceptos fundamentales. Madrid: Morata.

4. Köhler, W. (1972). Psicología de la forma. Su tarea y sus últimas experiencias. Madrid: Biblioteca Nueva.

5. Marx, K. y Engels, F. (1973). La ideología alemana. Barcelona: Progreso.

6. Maturana, H. y J. Luzoro. (1987). Diálogo con Humberto Maturana Romesín sobre Psicología. Rev. Chilena de Psicología. 9(1). 77-86.

7. Maturana, H. (1992). Emociones y Lenguaje en Educación y Política. Santiago de Chile: Pedagógicas Chilenas, S.A.

8. Maturana, H. (1994). Amor y juego. Fundamentos olvidados del ser humano. Santiago de Chile: Instituto de Psicoterapia.

9. Maturana, H. (1994). De máquinas y seres vivos. Autopoiesis: la organización de lo vivo. Santiago de Chile: Editorial Universitaria.

10. Maturana, H. (1994). El ojo del observador. Barcelona: Gedisa.

11. Maturana, H. (1995). El sentido de lo humano. Santiago de Chile: Dolmen

12. Maturana, H. (1996). Biología del emocionar y Alba Emoting. Santiago de Chile: Dolmen.
13. Maturana, H. (1996). Desde la biología a la psicología. Santiago de Chile: Pedagogía. Ed. Universitaria.

14. Maturana, H. (1996). La realidad: jobjetiva o construida? I: Fundamentos biológicos de la realidad. Barcelona: Anthropos.

15. Maturana, H. (1996). La realidad: ¿objetiva o construida? II: Fundamentos biológicos del conocimiento. Barcelona: Anthropos.

16. Maturana, H. (1999). Transformación en la convivencia. Santiago de Chile: Dolmen.

17. Maturana, H. (2003). Desde la biología a la psicología. Buenos Aires: Lumen.

18. Piaget, J. (1976a). Investigaciones sobre la contradicción. Madrid: Siglo XXI.

19. Piaget, J. (1976b). Psicología y pedagogía. México: Editorial Ariel.

20. Rosas, R. y Sebastián, C. (2010). Piaget, Vygotsky y Maturana: Constructivismo a tres voces. Buenos Aires: Aique.

21. Vygotsky, L. (1979). El desarrollo de los procesos psicológicos superiores. Barcelona: Crítica.

22. Vygotsky, L.S. (1981). Pensamiento y lenguaje. La Habana: Editorial Pueblo y Educación.

23. Wertsch, J. V. (1985). Vygotsky and the Social Formation of Mind. Cambridge, MA: Harvard University Press. 